\title{
Efficacy of an implanted automatic defibrillator which had induced atrial fibrillation
}

\author{
L JORDAENS, R HAMERLYNCK, D L CLEMENT \\ From the Departments of Cardiology and Cardiovascular Surgery, State University Ghent, Belgium
}

SUMMARY A 54 year old man with refractory life threatening ventricular tachycardia was given an automatic defibrillator. The initial system was a transvenous defibrillator coil electrode and this was later modified by implantation of two patch electrodes at thoracotomy.

The modified system successfully controlled ventricular tachycardia. On one occasion reversion of ventricular tachycardia by the defibrillator precipitated atrial fibrillation, a previously unreported side effect.

Internal defibrillators have been introduced to treat refractory and repeated ventricular tachycardia or fibrillation. We describe a patient in whom cardiac arrest was caused by ventricular fibrillation. A defibrillator was implanted four years after myocardial infarction.

\section{Case report}

In 1979 , when he was 50 , this man had had an inferoposterior myocardial infarction which was complicated with late ventricular tachycardia and cerebral embolism. Treatment with disopyramide was started and this was later changed to amiodarone ( $400 \mathrm{mg} /$ day) on the basis of Holter tape recordings which consistently showed frequent ventricular extrasystoles and couplets. He did not have angina pectoris or signs of left ventricular heart failure.

On 17 September 1983 he suddenly collapsed and cardiopulmonary resuscitation was carried out by bystanders. When the rescue team arrived ventricular fibrillation was detected and defibrillation was performed with $300 \mathrm{~J}$.

At examination blood pressure was $160 / 110 \mathrm{~mm}$ $\mathrm{Hg}$. A pansystolic murmur of grade II/VI intensity was recorded in the fourth intercostal space at the left sternal border. This had been present before the cardiac arrest. An electrocardiogram showed prominent depression of the J point with a horizontal ST segment in the precordial leads. The electrocardiogram subsequently showed sinus rhythm of 72 beats per minute with an axis in the frontal plane of

Requests for reprints to Dr L Jordaens, Akademisch Ziekenhuis, De Pintelaan 185, B-9000 Ghent, Belgium. $-10^{\circ}, \mathrm{QRS}$ width of $0 \cdot 10 \mathrm{~s}, \mathrm{Q}$ wave in lead III, and $Q R$ duration of $0.38 \mathrm{~s}$.

Serum electrolytes and creatinine and blood glucose concentrations were within the normal range. Serum aspartate aminotransferase activity was 113 IU/1 (normal 11-33 IU/1), lactic dehydrogenase was $425 \mathrm{IU} / \mathrm{l}$ (normal 200-400 IU/1), and creatine kinase was $51 \mathrm{IU} / 1$ (normal 0-150 IU/1) immediately after admission. Serum creatine kinase concentration reached a peak (268 IU/1) eight hours after admission but the MB fraction remained below $9 \mathrm{IU} / 1$. A chest $x$ ray film showed cardiomegaly with bilateral pulmonary venous congestion.

A coronary angiogram showed a proximal occlusion of the right coronary artery. An occlusion of the middle part of the circumflex artery was bridged by an extensive collateral circulation and there was a mild stenosis of the first diagonal artery of the left anterior descending artery. The inferior wall was akinetic. A left ventricular angiogram did not show any septal defect or mitral regurgitation. Ejection fraction, as measured with radionuclides was $42 \%$. Four weeks later a basic electrophysiological study was performed. Stimuli were delivered by a Jansen stimulator at a constant current of $<1 \mathrm{~mA}$ with pulse width of $2 \mathrm{~ms}$. Stimulation was applied above the right ventricular apex. Sustained hypotensive ventricular tachycardia with a cycle length of $240 \mathrm{~ms}$ was produced when double stimuli were applied to the heart in sinus rhythm. Rhythm was easily reproduced with double stimuli on a ventricular driven rhythm of 110 beats per minute.

In the subsequent serial drug testing procedure, intravenous procainamide on its own $(16 \mu \mathrm{g} / \mathrm{ml}$ and $6.5 \mu \mathrm{g} / \mathrm{ml}$ ) was ineffective in preventing stimulated 
tachycardia and it was still possible to induce ventricular tachycardia or fibrillation with the original stimulation protocol when the patient was on beta blockers or tocainide. After three weeks loading with oral amiodarone, ventricular fibrillation could still be easily induced by double stimuli on a paced rhythm of 90 beats per minute, and this situation was unchanged when amiodarone was given in combination with oral mexiletine. When amiodarone was given in combination with procainamide induction of fibrillation was slightly more difficult and double stimuli given on a basic rhythm of 110 beats per minute produced a hypotensive ventricular tachycardia of 150 beats per minute. Hence, the patient was started on oral procainamide $(1.5 \mathrm{~g}$ three times a day) in combination with amiodarone $(600 \mathrm{mg})$. This regimen was not considered to be effective because Holter tracings showed multiform ventricular premature beats and the patient had gastrointestinal symptoms and a productive cough.

On 11 April 1984 an automatic implantable defibrillator (AID-B ${ }^{R}$ ) (Intec Systems, Pittsburgh, USA) was implanted under general anaesthesia. A bipolar electrode was passed through the left cephalic vein and placed in the right ventricular apex. The amplitude of the $R$ wave was $12 \mathrm{mV}$. The Seldinger technique was used to place a classic defibrillator coil in the superior caval vein and a subxiphoid incision

\section{1}

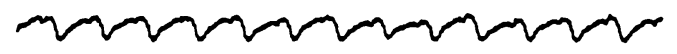

\section{5}

was made prepartory to positioning of a defibrillation patch.

Ventricular tachycardia with a cycle length of 320 ms could be induced with programmed electrical stimulation and could be stopped repeatedly with $5 \mathrm{~J}$ delivered through an external device (Fig. 1). In an attempt to induce ventricular fibrillation with programmed stimulation, however, ventricular tachycardia was induced by a burst of stimulation and when $20 \mathrm{~J}$ was delivered by the external cardioverter-defibrillator through the coil and patch the tachycardia rate was increased. A further shock of $30 \mathrm{~J}$ stopped the tachycardia. External cardioversion was needed to terminate ventricular fibrillation.

Anterolateral left thoracotomy was carried out so that a large defibrillation patch and a small defibrillation patch could be positioned in the pericardial space. The defibrillation threshold for ventricular fibrillation was $<20 \mathrm{~J}$. With the impulse generator in the abdominal pouch, fibrillation was produced by application of an alternating current. After a charge time of $8.2 \mathrm{~s}$, a first shock of $25.5 \mathrm{~J}$ was given after another 14 seconds. A third shock was needed to convert the ventricular fibrillation to sinus rhythm.

While the patient was still in hospital, an episode of ventricular tachycardia with loss of consciousness

aVF

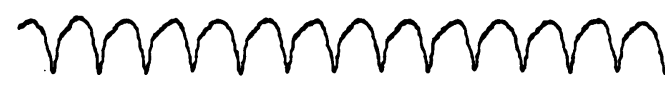

V6

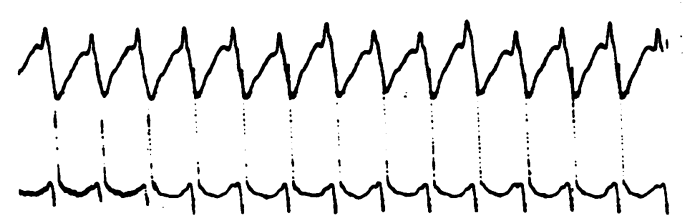

RV
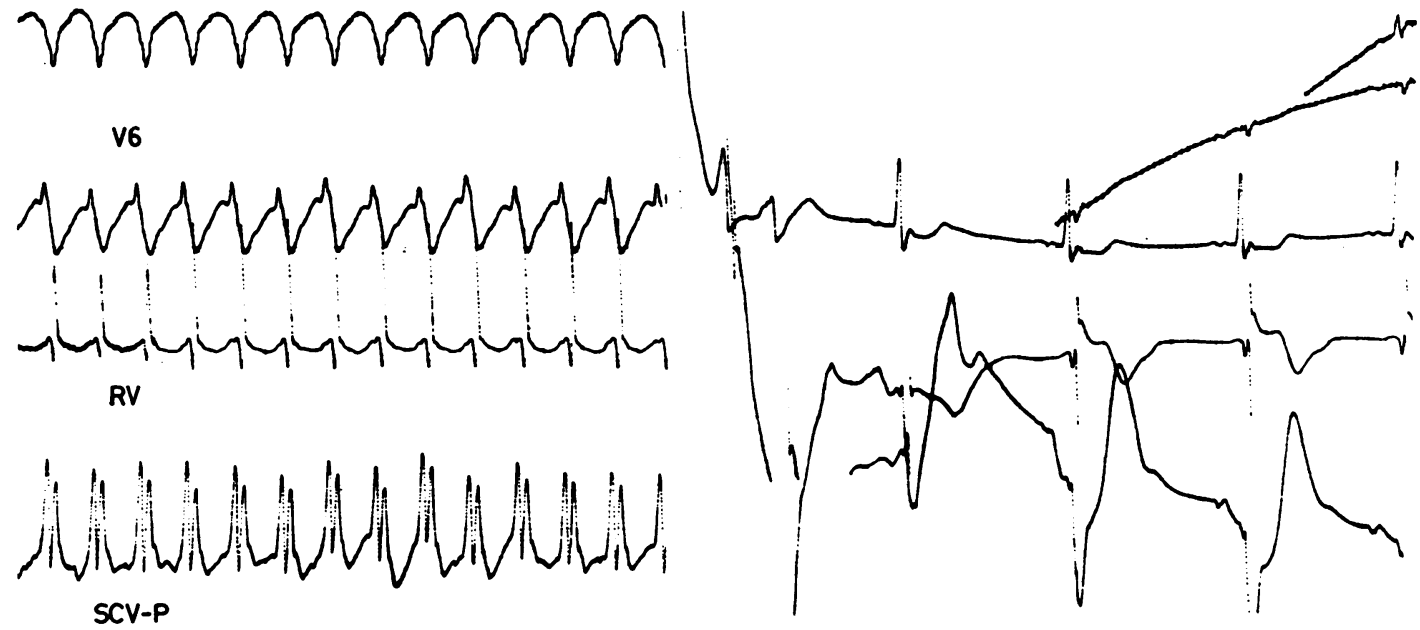

Fig. 1 Efficacy of defibrillator during monomorphous ventricular tachycardia. At implantation monomorphous tachycardia (cycle length $340 \mathrm{~ms}$ ) was interrupted by a $5 \mathrm{~J}$ shock between a coil in the superior caval vein and a patch in the pericardial space $(S C V-P)$. Ventricular fibrillation, however, was not terminated by $35 \mathcal{f}$ shock. $R V$, bipolar electrode in right ventricle. Paper speed was $25 \mathrm{~mm} / \mathrm{s}$. 
was terminated by the first shock. Termination of a recurrence required two internal shocks.

The patient was discharged in excellent condition, without antiarrhythmic drugs. Because we feared that the threshold of the defibrillator for ventricular fibrillation might be too high, a control electrophysiological study was performed on 25 May. Ventricular monomorphous tachycardia with cycle length of $330 \mathrm{~ms}$ was induced by double stimuli on a basic paced rhythm of 110 beats per minute. It was instantaneously detected and corrected by the first shock.

When a second episode of ventricular tachycardia terminated spontaneously a shock was given $290 \mathrm{~ms}$ after the $\mathbf{P}$ wave, and this produced long lasting atrial fibrillation and a ventricular rate of 100 beats per minute (Fig. 2). Ventricular fibrillation was produced by application of alternating current $(50 \mathrm{~Hz})$ for $2 \mathrm{~s}$ and the first shock of $25.5 \mathrm{~J}$ converted both rhythms to sinus rhythm. The patient was discharged after 24 hours. He is back at work.

\section{Discussion}

Ventricular fibrillation causes most sudden deaths from heart disease. ${ }^{1}$ The speed with which ventricular fibrillation can be terminated by defibrillation seems to be an important factor in determining the success of cardiopulmonary resuscitation, ${ }^{2}$ and an automatic implantable device that detects and corrects ventricular fibrillation immediately will be of considerable benefit in high risk patients. Such a device has been developed and implanted in more than 250 patients. $^{3-5}$

The clinical results are good, with a $25 \%$ decline in mortality at one year. ${ }^{5}$ These results were obtained in patients with older units and before the use of two patches or larger patches had been considered. $^{34}$ The 12 month survival rate in patients fitted with defibrillators is reported to be as high as $97.2 \%$ from ventricular tachycardia or fibrillation; it is $83.4 \%$ from all types of cardiovascular disease. These results show that implantable defibrillators are better than other forms of treatment for refractory ventricular rhythm disturbances. ${ }^{5}$

Much research has been done on the ideal positioning and size of the paddles and on the amount and character of the energy delivered to the myocardium. ${ }^{6}$ These studies led to the concept of a defibrillation threshold-that is the application of an appropriate amount of energy to the myocardium for a critical time. ${ }^{6}$ The sensing and defibrillation thresholds must be adjusted during implantation of the device. This influences the surgical technique and is relevant to previous and intended operations. ${ }^{7}$ If no other cardiovascular surgery is performed at the time of implantation, it is possible to insert the apical electrode through a small subxiphoid incision, as was our intention. ${ }^{8}$ This case report, however, confirms that some patients may show a high defibrillation threshold during the operation, or may even go into ventricular fibrillation when defibrillatory shocks are given. The conversion rate with the original spring patch defibrillator for mono-

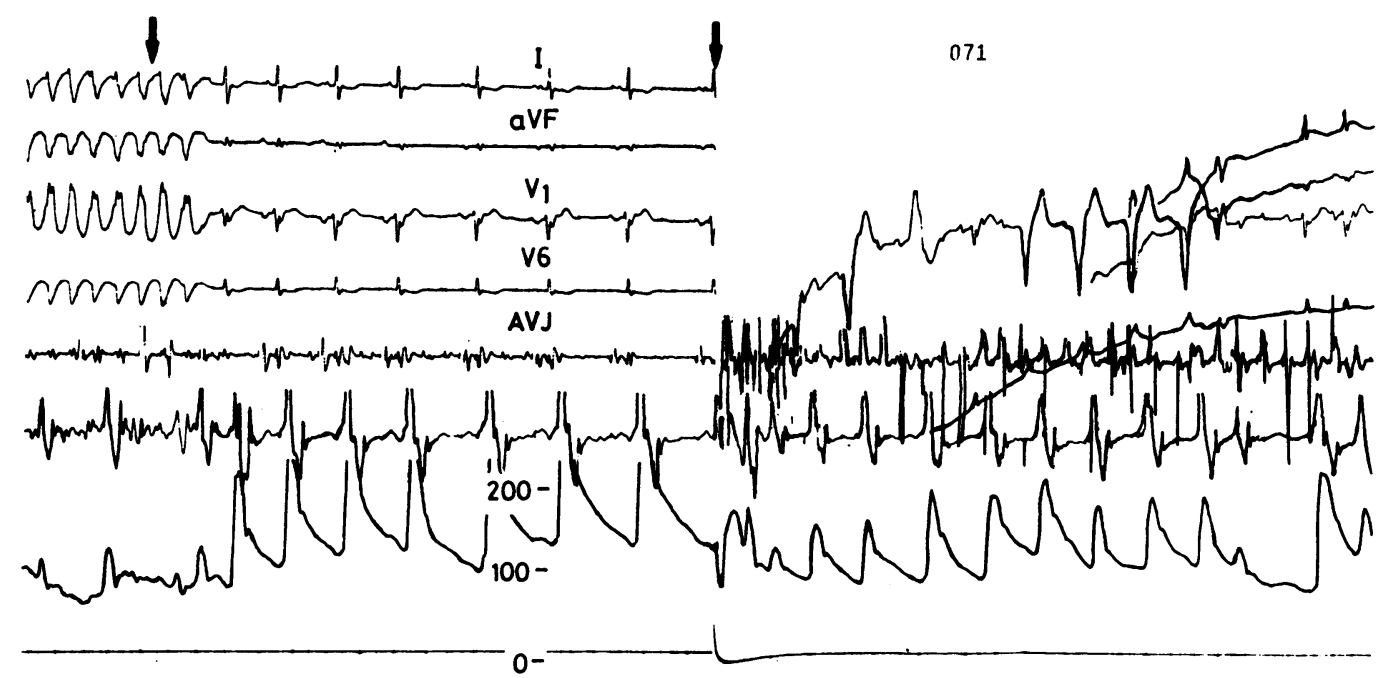

Fig. 2 Electrophysiological study after implantation of defibrillator. The induced regular ventricular tachycardia terminated spontaneously but was sensed (arrow at point of detection) by the defibrillator before sinus rhythm returned. An internal shock given $290 \mathrm{~ms}$ after the $P$ wave resulted in atrial fibrillation, as shown by the deviation of the atrioventricular junction ( $A V\}$ ) electrocardiogram. Lower tracing shows aortic pressure ( $\mathrm{mm} \mathrm{Hg}$ ). Paper speed was $25 \mathrm{~mm} / \mathrm{s}$. 
morphous ventricular tachycardia is $77-86 \%$, but the conversion rate is higher for polymorphous ventricular tachycardia and fibrillation when the size of the shock delivered to the myocardium is increased. ${ }^{7}$ About $10 \%$ of patients require two patches to produce a sufficiently low energy threshold. ${ }^{7}$ In these cases access through a subxiphoid incision is not adequate. Treatment of our patient with amiodarone may explain his high defibrillation threshold at operation. ${ }^{9}$

Acceleration of ventricular tachycardia with occurrence of fibrillation is a potential risk of all cardioversion systems, but this effect is less frequent with these devices than with burst pacing. ${ }^{10}$ Our case report shows that currently available defibrillators are capable of delivering a rescue shock because they can generate up to three more shocks after the initial shock. ${ }^{3}$

Control studies should be performed after implantation when it has not been possible to establish the efficacy or the correct sensing of clinical or induced arrhythmias during the implantation of the defibrillator, or when doubts about efficacy remain. We proposed a control study to our patient because cardioversion of ventricular fibrillation during implantation required three shocks, and two consecutive shocks were needed to convert clinical ventricular tachycardia to sinus rhythm. He might have required a high energy device. During the electrophysiological study correct sensing of both ventricular tachycardia and ventricular fibrillation were proved while the patient was under short general anaesthesia. We also found that when the device's capacitors were charged and the arrhythmia stopped spontaneously a shock was given when sinus rhythm returned. Synchronous shocks given during sinus tachycardia, supraventricular tachycardia, or atrial fibrillation are not known to have caused ventricular tachycardia or fibrillation. ${ }^{3}$ In our patient such a shock caused atrial fibrillation. It is clear, however, that because of synchronisation to ventricular rhythm during tachycardia the shock will often be dissociated from the atrial activity so that short lasting atrial arrhythmias may occur. ${ }^{3}$ The device has not been developed to treat atrial flutter or fibrillation, but these rhythm disturbances may trigger the defibrillator, and there is reversion to sinus rhythm in $50 \%$ of such episodes. ${ }^{11}$

\section{References}

1 Josephson ME, Horowitz LN, Spielman SR, Greenspan AM. Electrophysiologic and hemodynamic studies in patients resuscitated from cardiac arrest. $A m \mathcal{F}$ Cardiol 1979; 46: 948-55.

2 Eisenberg MS, Copass MK, Hallstrom AP, et al. Treatment of out-of-hospital cardiac arrests with rapid defibrillation by emergency medical technicians. $N$ Engl f Med 1980; 302: 1379-83.

3 Mirowski M, Reid PR, Winkle RA, et al. Mortality in patients with implanted automatic defibrillators. Ann Intern Med 1983; 98: 585-8.

4 Reid PR, Mower MM, Griffith SC, et al. Comparative effects on mortality of the first and second generation implantable defibrillators [Abstract]. Circulation 1984; 70 (suppl II): 101.

5 Mirowski M, Reid PR, Mower MM, et al. Clinical performance of the implantable cardioverterdefibrillator. PACE 1984; 7: 1345-50.

6 Schuder JC, Rahmoeller GA, Stoeckle H. Transthoracic ventricular defibrillation with triangular and trapezoidal waveforms. Circ Res 1966; 19: 689-94.

7 Winkle RA, Stinson EB, Bach SM Jr, Echt DS, Oyer $P$, Amstrong $K$. Measurement of cardioversion/ defibrillation thresholds in man by a truncated exponential waveform and an apical patch-superior vena caval spring electrode configuration. Circulation 1984; 69: 766-71.

8 Watkins L, Mirowski M, Mower MM, et al. Implantation of the automatic defibrillator: the subxiphoid approach. Ann Thorac Surg 1982; 34: 515-20.

9 Fogoros RN. Amiodaron-induced refractoriness to cardioversion. Ann Intern Med 1984; 100: 699-700.

10 Waspe LE, Kim SG, Matos JA, Fisher JD. Role of a catheter lead system for transvenous countershock and pacing during electrophysiologic tests: an assessment of the usefulness of catheter shocks for terminating ventricular tachyarrhythmias. Am $\mathcal{F}$ Cardiol 1983; 52: 477-84.

11 Reid PR, Mower MM, Mirowski M, et al. Correction of atrial tachyarrhythmias with the automatic implantable cardiovertor-defibrillator [Abstract]. Circulation 1983; 68 (suppl III): 1. 